\title{
Fluid Dynamics of Bacterial Turbulence
}

\author{
Jörn Dunkel, ${ }^{1}$ Sebastian Heidenreich, ${ }^{2}$ Knut Drescher, ${ }^{3}$ Henricus H. Wensink, ${ }^{4}$ Markus Bär, ${ }^{2}$ and Raymond E. Goldstein ${ }^{1}$ \\ ${ }^{1}$ DAMTP, Centre for Mathematical Sciences, University of Cambridge, Wilberforce Road, Cambridge CB3 OWA, United Kingdom \\ ${ }^{2}$ Physikalisch-Technische Bundesanstalt, Abbestrasse 2-12, 10587 Berlin, Germany \\ ${ }^{3}$ Departments of Molecular Biology and Mechanical and Aerospace Engineering, \\ Princeton University, Princeton, New Jersey 08544, USA \\ ${ }^{4}$ Laboratoire de Physique des Solides, Université Paris-Sud 11 and CNRS, Bâtiment 510, 91405 Orsay Cedex, France
}

(Received 21 February 2013; published 28 May 2013)

\begin{abstract}
Self-sustained turbulent structures have been observed in a wide range of living fluids, yet no quantitative theory exists to explain their properties. We report experiments on active turbulence in highly concentrated 3D suspensions of Bacillus subtilis and compare them with a minimal fourth-order vector-field theory for incompressible bacterial dynamics. Velocimetry of bacteria and surrounding fluid, determined by imaging cells and tracking colloidal tracers, yields consistent results for velocity statistics and correlations over 2 orders of magnitude in kinetic energy, revealing a decrease of fluid memory with increasing swimming activity and linear scaling between kinetic energy and enstrophy. The best-fit model allows for quantitative agreement with experimental data.
\end{abstract}

A series of experiments over the last decade [1-10] has shed light on generic ordering principles that appear to govern collective dynamics of living matter [11-15], from large-scale animal swarming $[1,2]$ to mesoscale turbulence in microbial suspensions [3-8] and microscale selforganization in motility assays $[9,10]$. Although very different in size and composition, these systems are often jointly termed "active" fluids, for which there is now a range of continuum theories [12,14-24]. From these have come important qualitative insights into instability mechanisms [13-16,21,25] driving dynamical pattern formation, but a quantitative picture remains inchoate; even for the simplest active (e.g., bacterial or algal) suspensions uncertainty remains about which hydrodynamic equations and transport coefficients [26,27] provide an adequate minimal description, due in large part to the inability of existing data to constrain the manifold parameters in these models. One approach to remedy this problem is to characterize collective turbulent dynamics of bacteria $[17,18]$ and other low Reynolds number swimmers, just as in high Reynolds number fluid turbulence, in terms of kinetic energy, mean squared vorticity (enstrophy) and spatiotemporal correlation functions, and to compare with an appropriate longwavelength theory (i.e., Navier-Stokes-type equations). We present such an analysis here, measuring collective behavior in dense suspensions of the bacterium Bacillus subtilis in comparison to predictions of a (fourth-order) continuum model for bacterial flow $[7,28]$.

Published by the American Physical Society under the terms of the Creative Commons Attribution 3.0 License. Further distribution of this work must maintain attribution to the author(s) and the published article's title, journal citation, and DOI.
Previous experimental studies of bacterial suspensions in open droplets [3,4,29,30], freestanding films [5,8,27,31], on surfaces $[6,32,33]$, or quasi-2D microfluidic chambers [7] focused separately on the bacterial and fluid components, leaving uncertain how accurately passive tracers $[34,35]$ reflect collective bacterial dynamics. The experiments reported here, performed in closed 3D microfluidic chambers, allowed near-simultaneous measurements of cell and tracer motion, and exploit a natural reduction in bacterial swimming activity due to oxygen depletion $[8,29,36]$ to obtain data spanning 2 orders of magnitude in fluid kinetic energy. Combined with extensive 3D numerical simulations of the model, this data allows robust parameter estimates. Quantitative agreement between experiment and theory suggests that this model presents a viable generalization of the Navier-Stokes equations to incompressible active fluids.

Wild-type strain 168 of $B$. subtilis has cigar-shaped cell bodies on average $0.8 \mu \mathrm{m}$ in diameter and $5 \mu \mathrm{m}$ long [7]. It was streaked on LB medium agar plates from frozen stocks. Colonies from these plates were used to inoculate overnight cultures in Terrific Broth (TB; Sigma), which were back-diluted 1:100 into $100 \mathrm{ml}$ of TB and kept on an orbital shaker at $37^{\circ} \mathrm{C}$ until reaching the middle of their exponential growth phase. These cultures were then concentrated $400 \times$ at $4000 \mathrm{~g}$ (final volume fraction $\sim 30 \%-40 \%$ ), and fluorescent microspheres (diameter $1 \mu \mathrm{m}, \mathrm{F}-8816$, Invitrogen) were added at a final concentration of $\sim 10^{9}$ beads $/ \mathrm{ml}$. The resulting suspensions were loaded into polydimethylsiloxane (PDMS) microfluidic devices, consisting of a series of cylindrical chambers (radius $750 \mu \mathrm{m}$, height $80 \mu \mathrm{m}$ ), connected by thin channels [7,37]. The inlet and outlet of the device were sealed with vacuum grease, and images were acquired in the $(x y)$ midplane of the chambers, $\approx 40 \mu \mathrm{m}$ above the bottom, using a 
Zeiss $40 \times($ NA 1.3) oil immersion objective and a highspeed camera at 40 fps (Fastcam SA-3, Photron). Movies were recorded in pairs for each field of view $(768 \times$ 800 pix; 1 pix $=0.36 \times 0.36 \mu \mathrm{m}^{2}$ ), one with bright-field illumination and one with fluorescence excitation by a $633 \mathrm{~nm}$ laser (B\&W Tek) at $\sim 20 \mathrm{~mW}$. These movies were taken immediately after each other with a $\sim 3 \mathrm{~min}$ time lag between subsequent pairs. During the $\sim 10 \mathrm{~min}$ imaging period for each device, the motility of $B$. subtilis cells decreased markedly due to oxygen depletion [29]. The experimental setup yields quasi-2D projected velocities of 3D suspension motion (see Fig. 1 and the Supplemental Material [38]). Data were analyzed under the assumption that the flow structures are isotropic, as verified by test measurements at different distances from the chamber bottom. Commercial particle image velocimetry (PIV) software (Dantec Flow Manager) was used to determine the bacterial flow velocity $\left(v_{x}, v_{y}\right)$ from bright-field images [Figs. 1(a) and 1(b)]. PIV data were corrected for systematic pixel-locking errors [30]. Data shown in Figs. 2 and 3 are based on seven movie segments (40 fps, each $50 \mathrm{~s}$ long) corresponding to seven different activity levels.

Adopting standard statistical measures from classical turbulence analysis, global bacterial flow properties were quantified by the in-plane kinetic energy $E_{x y}(t)=\left\langle\left(v_{x}^{2}+\right.\right.$ $\left.\left.v_{y}^{2}\right) / 2\right\rangle$ and in-plane enstrophy $\Omega_{z}(t)=\left\langle\omega_{z}^{2} / 2\right\rangle$, where

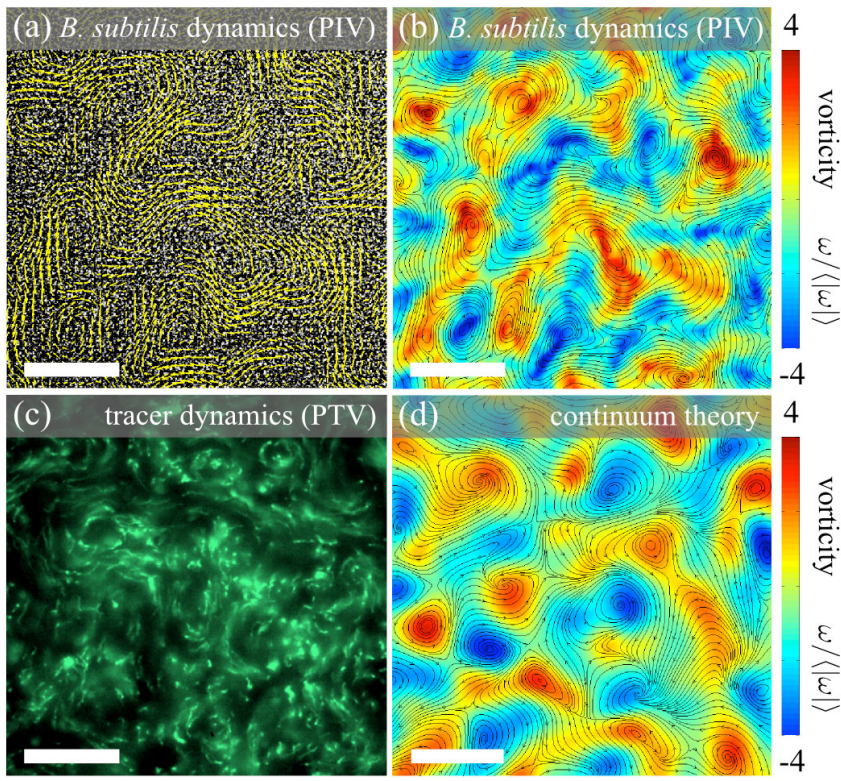

FIG. 1 (color online). Flow fields from experiments and simulations [38]. (a) Very dense homogeneous suspension of $B$. subtilis overlaid with the PIV flow field showing collective bacterial dynamics. Longest arrows correspond to velocity of $30 \mu \mathrm{m} / \mathrm{s}$. (b) Streamlines and normalized vorticity field determined from PIV data in (a). (c) Turbulent "Lagrangian" flow of fluorescent tracer particles (false-color) in the same suspension, obtained by integrating emission signals over 1.5 s. (d) Partial snapshot of a 2D slice from a 3D simulation of the continuum model (parameters in Table I). Scale bars $70 \mu \mathrm{m}$. $\omega_{z}=\partial_{x} v_{y}-\partial_{y} v_{x}$ is the vertical component of vorticity and $\langle\cdots\rangle$ is a spatial average. While $E_{x y}$ and $\Omega_{z}$ fluctuate, their time averages $\left(\bar{E}_{x y}, \bar{\Omega}_{z}\right)$ are approximately constant during the $50 \mathrm{~s}$ time interval used in the data analysis [Figs. 2(b) and 2(c)]. Over 2 orders of magnitude in energy [Fig. 2(d)] we observe the linear scaling $\bar{\Omega}_{z}=\bar{E}_{x y} / \Lambda^{2}$, with $\Lambda \approx 24 \mu \mathrm{m}$ being roughly one half of the typical vortex radius.

Probability distribution functions (PDFs) of the in-plane bacterial velocity are approximately Gaussian, with a slight broadening due to collective swimming [Fig. 2(a)]. The negative values of the equal-time spatial velocity correlation function [VCF; Fig. 3(a)] indicate the existence of vortices [4] (Fig. 1). The VCF is remarkably robust with respect to changes in the bacterial activity; in particular, the typical vortex radius $R_{v} \sim 40 \mu \mathrm{m}$, estimated from the first zero of the VCF, depends only weakly on the kinetic energy. This result is consistent with recent findings by Sokolov and Aranson [8] for freestanding films. The vortex

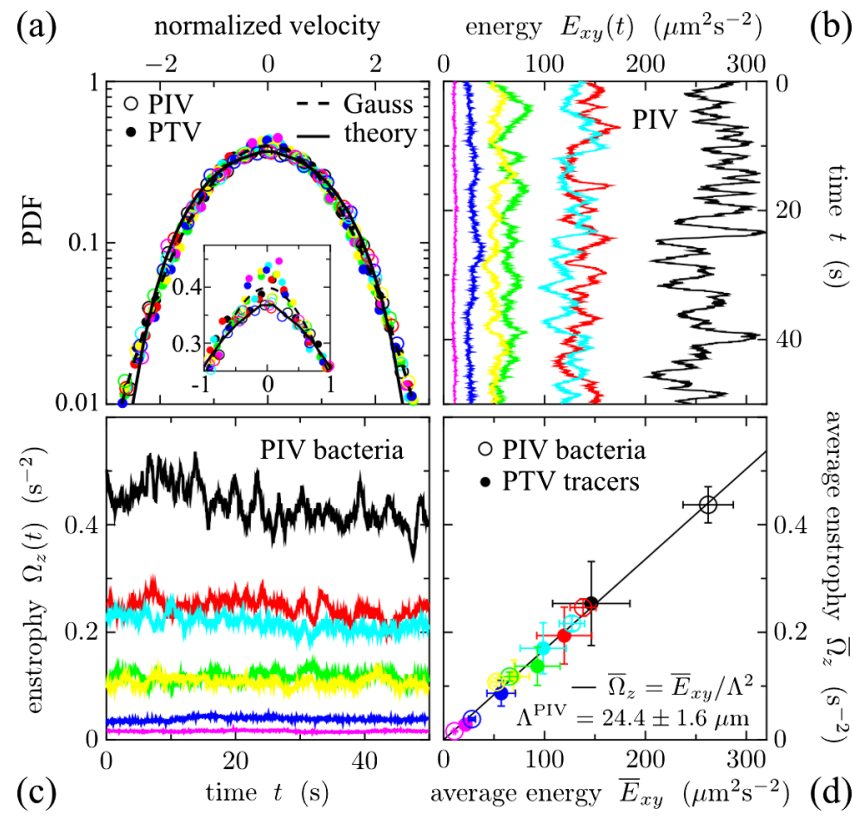

FIG. 2 (color online). Experimental results for bacterial and medium flows, color-coded for activity level. (a) PDFs of the Cartesian in-plane velocity components, normalized by their mean values and standard deviations, are approximately Gaussian (dashed) for both tracers and bacteria, with observable systematic deviations. The bacterial flow PDFs show slight broadening due to active swimming, which is well-reproduced by the model (1). By contrast, the PTV distributions exhibit higher peaks at small velocities (inset) due to accumulation of tracers near vortex centers. (b), (c) Mean kinetic energy and enstrophy of the in-plane bacterial flow components show moderate fluctuations during the data acquisition period, very similar to corresponding PTV data (not shown). (d) The time-averaged enstrophy scales linearly with the time-averaged energy. Open circles are averages of the curves in (b), (c). Error bars indicate temporal standard deviations. 


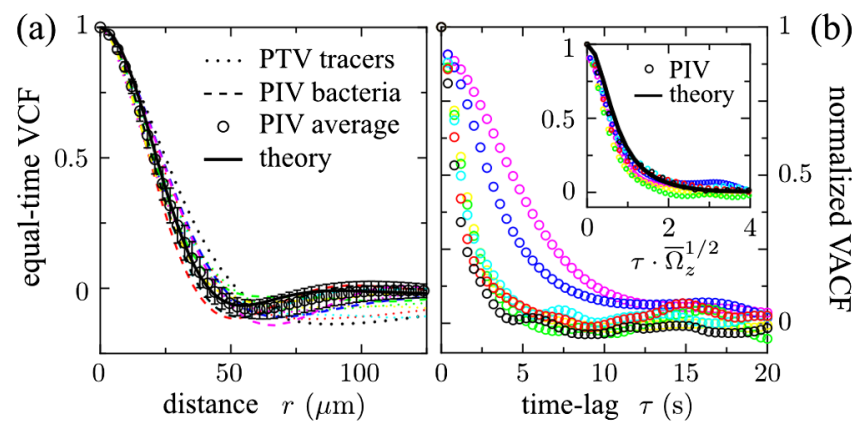

FIG. 3 (color online). Correlation functions for solvent (PTV) and bacterial (PIV) flow at different energies and best-fit continuum theory (see Table I), using the same colors as in Fig. 2. (a) Both PIV and PTV data indicate a characteristic vortex radius $R_{v} \sim 40 \mu \mathrm{m}$. The decay of spatial correlations at small $r$ depends only weakly on the activity level. (b) Velocity autocorrelation functions of the bacterial flow collapse when the time lag $\tau$ is rescaled (inset) by the enstrophy time scale $\bar{\Omega}_{z}^{-1 / 2}$. Since $\bar{\Omega}_{z} \propto \bar{E}_{x y}$ [Fig. 2(d)], this implies that the higher the bacterial activity the shorter the flow memory.

size in 3D is roughly five times larger than for quasi-2D turbulence in thin microfluidic chambers [7], where bacterial swimming and hydrodynamic interactions are suppressed by the nearby no-slip boundaries [37,39]. Unlike the spatial VCF, the two-time velocity auto-correlation function (VACF) varies systematically with energy or vorticity [Fig. 3(b)], but they collapse when plotted as functions of the dimensionless lag-parameter $\tau \Omega_{z}^{1 / 2}$ [inset of Fig. 3(b)], implying that the higher the activity the shorter the memory of the bacterial fluid. Generally, the statistics of 3D bacterial turbulence differ strongly from conventional 3D Navier-Stokes turbulence [40,41], as bacteria inject energy on the smallest scales, inducing an "upward" energy cascade toward larger length scales.

We infer the flow of the solvent medium from particle tracking velocimetry (PTV) analysis of the fluorescence images, which only show the tracer particles, assuming that they are passively advected. Data shown in Figs. 2 and 3 are based on 7 movies (40 fps, length $100 \mathrm{~s}$ ) at different activities. Trajectories of individual tracer particles were found with a custom algorithm which, depending on seeding density and tracer dynamics, was able to identify up to $10^{4}$ in-plane tracks, the longest typically lasting 5-8 s. The effective sample size was insufficient to determine reliably the tracer VACFs, but did yield global flow properties, velocity histograms and equal-time VCFs. The velocity PDFs, calculated directly from individual tracer velocities, are approximately Gaussian with a peak at small velocities from tracer accumulation near the vortex centers [Fig. 2(a)].

Estimates from PTV for the medium VCF and enstrophy were obtained by interpolating tracer velocities on a $450 \times 450$ pix subwindow in the center of the imaging plane using MATLAB's Delaunay triangulation with a lattice spacing $\Delta=90 \sqrt{\mathrm{pix} / N_{f}}$, where $N_{f}$ is the mean number of tracers detected per frame. The accuracy of this reconstruction procedure is controlled by the tracer concentration, which was kept low to limit effects on the bacteria motion and to avoid tracking ambiguities (typically $N_{f} \in$ $[47,144]$ for data shown in Figs. 2 and 3). As a result, the uncertainties for the PTV data are considerably larger than for PIV data [see Fig. 2(d)]. The interpolated tracer flow fields were used to estimate the kinetic energy $E_{x y}$, enstrophy $\Omega_{z}$, and spatial correlation functions of the in-plane medium flow components. In agreement with the PIV results for the bacterial flow, we find again a linear enstrophy-energy relation [Fig. 2(d)] and comparable vortex radii, using the first zero of the VCF as an estimate [Fig. 3(a)]. We may therefore conclude that, at our very high bacterial concentrations, solvent and bacterial flow statistics become tightly linked.

We now examine how these data compare to predictions of a theory of active fluids introduced recently $[7,28]$. This minimal continuum model assumes that, at high concentrations, the bacterial flow due to swimming and advection can be described by a single velocity field $\boldsymbol{v}(t, \boldsymbol{x})$ and a pressure $p(t, \boldsymbol{x})$. The theory accounts, phenomenologically, for the most relevant physical effects: incompressibility, local jet-formation by polar alignment, nematic interactions, and stress-induced instability, represented by higher-order spatial derivatives arising from a longwavelength expansion of the effective stress-tensor [28]. The field dynamics is governed by the incompressibility condition $\nabla \cdot \boldsymbol{v}=0$ and

$$
\begin{aligned}
\left(\partial_{t}+\lambda_{0} \boldsymbol{v} \cdot \nabla\right) \boldsymbol{v}= & -\nabla p+\lambda_{1} \nabla \boldsymbol{v}^{2}-\beta\left(\boldsymbol{v}^{2}-v_{0}^{2}\right) \boldsymbol{v} \\
& +\Gamma_{0} \nabla^{2} \boldsymbol{v}-\Gamma_{2}\left(\nabla^{2}\right)^{2} \boldsymbol{v} .
\end{aligned}
$$

Equation (1) extends the incompressible Toner-Tu theory $[14,19,42]$ with a fourth-order term as in the SwiftHohenberg equation [43]. The parameter $\lambda_{0}$ describes advection and nematic interactions, and $\lambda_{1}$ an active pressure contribution [28]. For pusher swimmers [37] like $B$. subtilis, general considerations of hydrodynamic [44] and nematic stresses $[28,45]$ suggest that $\lambda_{0} \geq 1$ and $\lambda_{1} \simeq$ $\left(\lambda_{0}-1\right) / 3 \geq 0$ in $3 \mathrm{D}$. The $\left(\beta, v_{0}\right)$ terms correspond to a quartic Landau-type velocity potential $[14,19,42]$ and are physically motivated by the observation of extended jetlike streaming regions in $B$. subtilis suspensions at intermediate concentrations [30]. The parameter $v_{0}$ defines the collective speed that would be achieved if all bacteria were to move in the same direction. When $\beta \neq 0$ the model does not conserve momentum or energy, as it describes exclusively the bacterial flow component, which may exchange energy and momentum with the solvent. The nonlocal $\left(\Gamma_{0}\right.$, $\Gamma_{2}$ ) terms encode passive and active stresses due to hydrodynamic and steric interactions. For $\lambda_{0}=1, \lambda_{1}=\beta=$ $\Gamma_{2}=0$ and $\Gamma_{0}>0$, the model reduces to the incompressible Navier-Stokes equation. A detailed stability analysis 

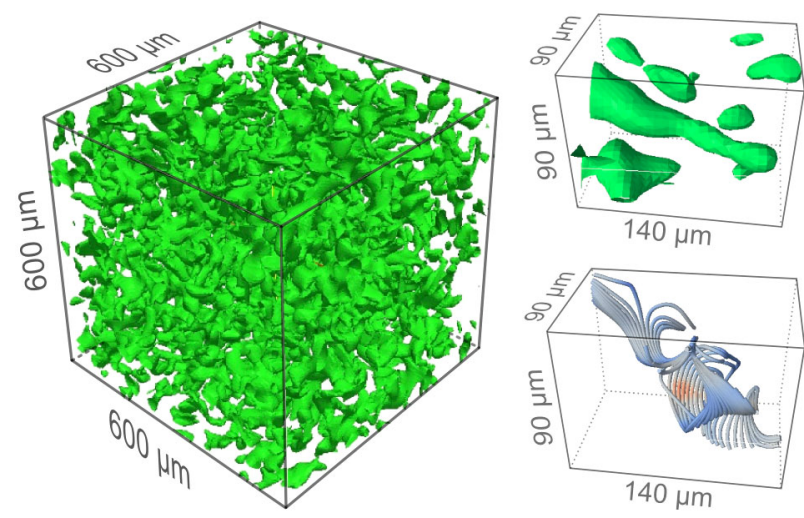

FIG. 4 (color online). Isoenergy surfaces $\left(E=1.7 v_{0}^{2}\right)$ and selected stream tubes from the best-fit 3D simulation (visualized with PARAVIEW) indicate a typical vortex length scale and extended bandlike regions corresponding to coaligned bacterial jets. See Supplemental Material [38] for a movie.

[28] shows that when $\lambda_{0} \neq 0, \beta>0, v_{0}>0, \Gamma_{2}>0$, and $\Gamma_{0}<0$, this is one of the simplest vector models to describe phenomenologically the formation of jets and turbulent vortices in quasi-incompressible active suspensions. Very recently, the 2D version of Eq. (1) has been shown to provide a quantitative mean field description of bacterial mesoscale turbulence in quasi-2D suspensions [7]. Its applicability to the physically more relevant 3D case is first explored here.

We simulated Eq. (1) in 3D with periodic boundary conditions using a pseudospectral operator-splitting algorithm $[46,47]$ and a pressure correction subroutine to ensure incompressibility [7,28]. Simulation grids ranged from $128^{3}$ lattice points for parameter prescreening to $256^{3}$ for statistical analysis. Numerical stability of the solver was verified for a wide range of parameters and space-time discretizations. All simulations were initiated with randomly chosen velocities. Figure 4 shows structure formation in a typical simulation domain.

Since in 3D we have $\lambda_{1} \simeq\left(\lambda_{0}-1\right) / 3$ [28], Eq. (1) has essentially five free parameters $\left(\lambda_{0}, \beta, v_{0}, \Gamma_{0}, \Gamma_{2}\right)$. Two of those can be eliminated by choice of appropriate length and time units. We adopt a natural unit system such that the vortex wavelength scale $\Lambda_{\Gamma}=2 \pi \sqrt{\Gamma_{2} /\left(-\Gamma_{0}\right)}=2 \pi$ and $v_{0}=1$. In our simulations, the box length is fixed as $L=12 \Lambda_{\Gamma}$, corresponding to approximately twice the experimental field of view, and the time step as $\Delta t=$ $0.05 \Lambda_{\Gamma} /\left(2 \pi v_{0}\right)$. To estimate the three remaining parameters $\left(\lambda_{0}, \beta, \Gamma_{0}\right)$, we note that $\Gamma_{0}$ and $\Gamma_{2}$ define a typical vortex speed $V_{\Gamma}=\sqrt{-\Gamma_{0}^{3} / \Gamma_{2}}$. In the turbulent regime, it is plausible that $V_{\Gamma}$ is smaller than but close to $v_{0}$, i.e., $V_{\Gamma}=$ $\zeta v_{0}$ where $\zeta \lesssim 1$. Furthermore, for pushers, the dimensionless parameter $\lambda_{0}$ should be larger than 1 , but smaller than for quasi-2D suspensions [7], since nematic (steric) stresses can be more easily avoided in 3D; we infer $\lambda_{0} \sim 2$. Finally, the acceleration time scale $\tau_{0}=\left(\beta v_{0}^{2}\right)^{-1}$ should
TABLE I. Parameters of the best-fit continuum model. To match a specific experiment, one must merely adjust the physical value of $v_{0}$ by equating $\bar{E}_{x y}=0.54 v_{0}^{2}$ to the corresponding kinetic energy value in Fig. 2(d).

\begin{tabular}{lcc}
\hline \hline Model parameter & In rescaled units & In physical units \\
\hline$\Lambda_{\Gamma}=2 \pi \sqrt{\Gamma_{2} /\left(-\Gamma_{0}\right)}$ & $2 \pi$ & $\sim 50 \mu \mathrm{m}$ \\
$v_{0}$ & 1 & $3-22 \mu \mathrm{m} / \mathrm{s}$ \\
$\lambda_{0}$ & 1.7 & 1.7 \\
$V_{\Gamma}=\sqrt{-\Gamma_{0}^{3} / \Gamma_{2}}$ & 0.9 & $0.9 v_{0}$ \\
$\beta$ & 0.1 & $1.3 \times 10^{-2}\left(v_{0} \mu \mathrm{m}\right)^{-1}$ \\
$\bar{E}_{x y}$ & 0.54 & $0.54 v_{0}^{2}$ \\
\hline \hline
\end{tabular}

be of the order of the vortex time scale $\Lambda_{\Gamma} / V_{\Gamma}$. Using these estimates as initial values in a systematic parameter scan, and by comparing with the bacterial PIV data, we obtained the best-fit parameters in Table I. Generally, the VCFs and VACFs respond sensitively to parameter variations in the simulations, suggesting that the estimates in Table I are accurate within 10\%-15\% for quasi-incompressible $B$. subtilis suspensions. As an independent cross-check, we computed $\Lambda=\left(\bar{E}_{x y} / \Omega_{z}\right)^{1 / 2}$ from the best-fit simulation using $\Lambda_{\Gamma} \sim 50 \mu \mathrm{m}$ and found $\Lambda \sim 29 \mu \mathrm{m}$ which compares well with the experimental PIV value in Fig. 2(d). We stress that the conserved form of the bacterial velocity PDFs [Fig. 2(a)], VCFs, and VACFs (Fig. 3) implies that all our experiments can be fitted by a single set of rescaled parameters $\left(\lambda_{0}, \beta, \Gamma_{0}\right)$, as it suffices to adjust the physical values of $v_{0}$ and $\Lambda_{\Gamma}$ to match the kinetic energy and vortex length at a given bacterial activity level. As evident from the flow patterns in Fig. 1 and from the solid curves in Figs. 2(a) and 3, the best-fit parameters yield good qualitative and quantitative agreement with the experiments.

For incompressible "passive" fluids, that are governed by the Navier-Stokes equations, transport parameters have of course been measured for a wide range of materials [48]. In contrast, quantitative theories of even the simplest active fluids have been lacking. We have shown here that the minimal fourth-order vector model $[7,28]$ in Eq. (1) reproduces the main statistical features of self-sustained 3D bulk turbulence in concentrated bacterial suspensions, suggesting that this theory is a viable candidate for the quantitative description of incompressible active fluids. Due to the close correlation between bacterial and medium (tracer) flow observed in our experiments, we expect that this generic model will be useful in a wide range of future applications, in particular for predicting the effects of confining geometries on collective microbial dynamics $[49,50]$ and for understanding the anomalous viscosities of active fluids [26,27].

The authors would like to thank Igor Aranson, Vasily Kantsler, Sabine Klapp, Hartmut Löwen, Cristina Marchetti, Lutz Schimanksy-Geier, Holger Stark, Hugo Wioland, Francis Woodhouse, and Julia Yeomans for helpful discussions. This work was supported by the Deutsche 
Forschungsgemeinschaft, Grant No. GRK1558 (M. B. and S. H.), the Human Frontier Science Program (K. D.), and the European Research Council, Advanced Investigator Grant No. 247333 (J. D. and R. E. G.). J. D. and S. H. contributed equally to the Letter and are joint first authors.

[1] Y. Katz, C. C. Ioannou, K. Tunstro, C. Huepe, and I. D. Couzin, Proc. Natl. Acad. Sci. U.S.A. 108, 18720 (2011).

[2] A. Cavagna, A. Cimarelli, I. Giardina, G. Parisi, R. Santagati, F. Stefanini, and M. Viale, Proc. Natl. Acad. Sci. U.S.A. 107, 11865 (2010).

[3] J. O. Kessler and M. Wojciechowski, Collective Behavior and Dynamics of Swimming Bacteria (Oxford University Press, Oxford, England, 1997), pp. 417-450.

[4] C. Dombrowski, L. Cisneros, S. Chatkaew, R.E. Goldstein, and J. O. Kessler, Phys. Rev. Lett. 93, 098103 (2004).

[5] A. Sokolov, I.S. Aranson, J. O. Kessler, and R.E. Goldstein, Phys. Rev. Lett. 98, 158102 (2007).

[6] H. P. Zhang, A. Be'er, E.-L. Florin, and H. L. Swinney, Proc. Natl. Acad. Sci. U.S.A. 107, 13626 (2010).

[7] H. H. Wensink, J. Dunkel, S. Heidenreich, K. Drescher, R. E. Goldstein, H. Löwen, and J. M. Yeomans, Proc. Natl. Acad. Sci. U.S.A. 109, 14308 (2012).

[8] A. Sokolov and I. S. Aranson, Phys. Rev. Lett. 109, 248109 (2012).

[9] V. Schaller, C. Weber, C. Semmrich, E. Frey, and A. R. Bausch, Nature (London) 467, 73 (2010).

[10] Y. Sumino, K. H. Nagai, Y. Shitaka, D. Tanaka, K. Yoshikawa, H. Chaté, and K. Oiwa, Nature (London) 483, 448 (2012).

[11] M.F. Copeland and D. B. Weibel, Soft Matter 5, 1174 (2009).

[12] D. L. Koch and G. Subramanian, Annu. Rev. Fluid Mech. 43, 637 (2011).

[13] T. Vicsek and A. Zafeiris, Phys. Rep. 517, 71 (2012).

[14] S. Ramaswamy, Annu. Rev. Condens. Matter Phys. 1, 323 (2010).

[15] M. C. Marchetti, J.-F. Joanny, S. Ramaswamy, T. B. Liverpool, J. Prost, M. Rao, and R. A. Simha, arXiv: $1207.2929 v 1$.

[16] K. Kruse, J.-F. Joanny, F. Jülicher, J. Prost, and K. Sekimoto, Phys. Rev. Lett. 92, 078101 (2004).

[17] E. Lauga and R. E. Goldstein, Phys. Today 65, No. 9, 30 (2012).

[18] C. W. Wolgemuth, Biophys. J. 95, 1564 (2008).

[19] J. Toner and Y. Tu, Phys. Rev. E 58, 4828 (1998).

[20] A. Baskaran and M. C. Marchetti, Proc. Natl. Acad. Sci. U.S.A. 106, 15567 (2009).

[21] D. Saintillan and M. Shelley, Phys. Fluids 20, 123304 (2008).

[22] A. Peshkov, I. S. Aranson, E. Bertin, H. Chaté, and F. Ginelli, Phys. Rev. Lett. 109, 268701 (2012).

[23] T. Brotto, J.-B. Caussin, E. Lauga, and D. Bartolo, Phys. Rev. Lett. 110, 038101 (2013).
[24] S.P. Thampi, R. Golestanian, and J.M. Yeomans, arXiv:1302.6732v1.

[25] R. Grossmann, L. Schimansky-Geier, and P. Romanczuk, arXiv:1301.5890v2.

[26] S. Rafai, L. Jibuti, and P. Peyla, Phys. Rev. Lett. 104, 098102 (2010).

[27] A. Sokolov and I.S. Aranson, Phys. Rev. Lett. 103, 148101 (2009).

[28] J. Dunkel, S. Heidenreich, M. Bär, and R. E. Goldstein, New J. Phys. 15, 045016 (2013).

[29] I. Tuval, L. Cisneros, C. Dombrowski, C. W. Wolgemuth, J. O. Kessler, and R. E. Goldstein, Proc. Natl. Acad. Sci. U.S.A. 102, 2277 (2005).

[30] L.H. Cisneros, J.O. Kessler, S. Ganguly, and R.E. Goldstein, Phys. Rev. E 83, 061907 (2011).

[31] K.-A. Liu and L. I, Phys. Rev. E 86, 011924 (2012).

[32] H. P. Zhang, A. Be'er, R.S. Smith, E. Florin, and H. L. Swinney, Europhys. Lett. 87, 48011 (2009).

[33] F. Peruani, J. Starruß, V. Jakovljevic, L. SogaardAndersen, A. Deutsch, and M. Bär, Phys. Rev. Lett. 108, 098102 (2012).

[34] X.-L. Wu and A. Libchaber, Phys. Rev. Lett. 84, 3017 (2000).

[35] T. Ishikawa, N. Yoshida, H. Ueno, M. Wiedeman, Y. Imai, and T. Yamaguchi, Phys. Rev. Lett. 107, 028102 (2011).

[36] C. Douarche, A. Buguin, H. Salman, and A. Libchaber, Phys. Rev. Lett. 102, 198101 (2009).

[37] K. Drescher, J. Dunkel, L. H. Cisneros, S. Ganguly, and R. E. Goldstein, Proc. Natl. Acad. Sci. U.S.A. 108, 10940 (2011).

[38] See Supplemental Material at http://link.aps.org/ supplemental/10.1103/PhysRevLett.110.228102 for videos.

[39] S. E. Spagnolie and E. Lauga, J. Fluid Mech. 700, 105 (2012).

[40] U. Frisch, Turbulence (Cambridge University Press, Cambridge, England, 2004).

[41] H. Xu, M. Bourgoin, N. T. Ouellette, and E. Bodenschatz, Phys. Rev. Lett. 96, 024503 (2006).

[42] J. Toner, Y. Tu, and S. Ramaswamy, Ann. Phys. (Amsterdam) 318, 170 (2005).

[43] J. Swift and P.C. Hohenberg, Phys. Rev. A 15, 319 (1977).

[44] T. J. Pedley, Exp. Mech. 50, 1293 (2010).

[45] A. Baskaran and M. C. Marchetti, Phys. Rev. E 77, 011920 (2008).

[46] D. Gottlieb and S. A. Orszag, Numerical Analysis of Spectral Methods: Theory and Applications (SIAM, Montpelier, VT, 1977).

[47] J. Pedrosa, M. Hoyuelos, and C. Martel, Eur. Phys. J. B 66, 525 (2008).

[48] C. L. Yaws, Handbook of Transport Property Data: Viscosity, Thermal Conductivity, and Diffusion Coefficients of Liquids and Gases (Institution of Chemical Engineers, London, 1995).

[49] F. G. Woodhouse and R. E. Goldstein, Phys. Rev. Lett. 109, 168105 (2012).

[50] M. Ravnik and J.M. Yeomans, Phys. Rev. Lett. 110, 026001 (2013). 